\title{
Slip flow past a gas-liquid interface with embedded solid particles
}

\author{
A. Vidal and L. Botto $\dagger$ \\ School of Engineering and Materials Science, Queen Mary University of London, Mile End \\ Road, London E1 4NS, UK
}

(Received xx; revised xx; accepted xx)

We simulate shear flow past a stationary monolayer of spherical particles embedded in a flat gas-liquid interface. This problem is relevant to the understanding of the microhydrodynamics of particle-laden interfacial structures, including particle-laden drops, bubbles and foams. The combination of the free-shear condition at the gas-liquid interface and the no-slip condition at the particle surfaces gives rise to a velocity slip at the particleladen interface. We study the characteristics of the flow near the monolayer, focusing on slip velocity, slip length and interfacial shear stress. Two microstructures are compared: a square array, and a reticulated array mimicking a percolating network of aggregated particles. We demonstrate that the scaling laws for the dependence of the slip length on solid area fraction developed for flow past superhydrophobic microstructured surfaces apply to the case of interfacial particles. The calculated slip lengths are in general smaller that those reported for microstructured superhydrophobic surfaces. This differences, which is due to the significant protrusion of the spherical particles in the liquid, can be accounted for in the case of the square array by an approximate argument. For a given area fraction, the reticulated array yields a larger slip length than the square array. We analyse the hydrodynamic forces acting on the particles, and the corresponding tangential stress exerted by the bulk "subphase".

\section{Introduction}

In applications and natural settings gas-liquid interfaces are often found covered with particulate material. Fouling of gas-liquid interfaces can occur in unsaturated porous media or environmental bubbly flows, owing to the presence of fine grains and biocolloids that adhere to the fluid interface (Shang et al. 2009; Weber et al. 1983). In applications, rigid particles or globular proteins are often added as surface active agents to change the mechanical properties of the interface or induce stabilisation against coalescence (Tambe \& Sharma 1994; Binks 2002; Stancik et al. 2004).

As for molecular surfactants, the presence of the embedded solid particles alters the boundary conditions at a fluid interface (for experimental evidence see e.g. Hunter et al. (2008) and Kotula \& Anna (2012); for a theoretical analysis, see Deemer \& Slattery (1978)). These boundary conditions for the bulk fluid are to be applied to the particleladen fluid interface, i.e. the composite interface formed by the particles and the fluid interface in which the particles are embedded. In the absence of mass transfer effects, the no-penetration condition at the particle-laden interface is expected to hold with good accuracy. However, the boundary condition for the velocity tangential to the particleladen fluid interface must be modified to account for the additional resistance caused by the presence of the particles to the motion of the adjacent fluid layers. This additional

$\dagger$ Email address for correspondence: 1.botto@qmul.ac.uk 

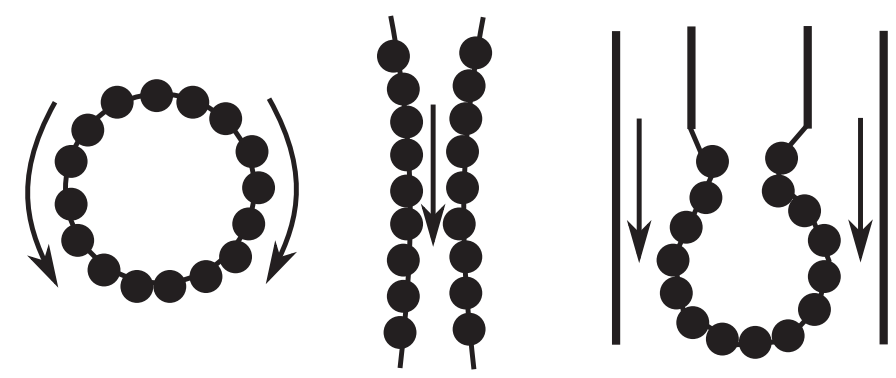

Figure 1: Examples of flow problems involving gas-liquid particle-laden interfaces where significant particle-fluid velocity slip is expected: from left to right, a rising particlecoated bubble (Weber et al. 1983), liquid drainage in particle-laden thin films (Bournival et al. 2015; Stancik et al. 2004; Hunter et al. 2008), formation of particle-coated bubbles in a microfluidic device (Subramaniam et al. 2005; Kotula \& Anna 2012).

resistance is expected to be particularly significant when a significant velocity difference occurs between the particle-laden fluid interface and the adjacent fluid (figure 1).

In this paper we simulate shear flow past a gas-liquid interface containing a monolayer of spherical particles, for the case in which the particle-laden fluid interface is flat and the monolayer is stationary (or moving with constant velocity if a change of reference is accounted for). All the simulations are carried out in the Stokes flow limit. The simulation results allow to gain insights into the dependence of the slip length parameter appearing in a partial slip boundary condition for the particle-laden interface on the macroscopic flow variables and particle distribution. Such boundary condition could be applied to problems related to froth flotation (Subrahmanyam \& Forssberg 1988), solid stabilised foams and emulsions (Horozov 2008; Martinez et al. 2008) and spray-drying (Tsapis et al. 2005).

Recently, the statics and dynamics of particles embedded in fluid interfaces has been subject to increasing interest. Singh \& Joseph (2005) studied the equilibrium condition for particles supported by surface tension at a horizontal fluid interface, as a function of particle weight and contact angle. Under the effect of gravity particles induce a distortion of the fluid interface whose amplitude is proportional to the magnitude of the particle weight. For particles in the size range of typical colloids $(a<10 \mu m)$, the particle weight is orders of magnitude smaller than the capillary force on the particle. As a consequence the interface around the particle can be considered locally flat and unaffected by the gravitational force acting on the particle. This notion can be generalised to non-horizontal particle-laden interfaces. For curved particle-laden fluid interfaces, the composite interface can be considered locally flat if the particle radius is small in comparison to the radius of curvature of the particle-laden interface. For a locally flat interface, the degree of protrusion of a solid particles in the liquid phase is only a function of the contact angle $\theta_{c}$ and the particle radius (Rapacchietta \& Neumann 1977).

The drag forces on single spheres embedded in fluid-fluid interfaces has been studied by several authors. For gas-liquid interfaces, the drag force is a monotonic function of the degree of protrusion of the particle in the liquid phase (Petkov et al. 1995; Danov et al. 2000; Fischer et al. 2006). When $\theta_{c}=90^{\circ}$, owing to symmetry, the drag on an isolated sphered in a uniform flow is exactly half the Stokes drag for a fully-immersed sphere. A particle embedded in a gas-liquid interface and subject to a shear flow will also experience a hydrodynamic torque and may rotate (Pozrikidis 2007). However, effects due to rotation, which depend on the shear rate, are expected to be subdominant with 
respect to those due to relative translation between the particle and the surrounding fluid; for small particles contact line pinning may prevent rotation completely, and this is a situation often found in practice (Dörr et al. 2016). Only a few studies have investigated the hydrodynamics of multiple interfacial particles. These studies are typically concerned with the dynamics of small clusters of particles (Singh \& Joseph 2005; Dani et al. 2015), or to the macroscopic effect of the collective motion of many particles on the dynamics of liquid-liquid interfacial structures (Frijters et al. 2012). To the best of our knowledge, the interfacial drag on multiple particles subject to shear flow and the characteristics of slip flow for this flow configuration have not been considered in the literature.

The flow past a stationary monolayer of spheres embedded in a gas-liquid interface bears obvious similarities to flow past a microstructured superhydrophobic surface. In both cases one can define a composite surface composed of free-slip and no-slip "patches". From the point of view of continuum modelling, i.e. considering flow variables on a scale much larger than the particles, the boundary condition at such composite surface is expected to be a linear combination of the boundary conditions that are appropriate for the solid and fluid regions. Composite free-slip/no-slip interfaces indeed have been successfully modelled through a Navier slip boundary condition

$$
\lambda\langle\dot{\gamma}\rangle_{s}=\langle u\rangle_{s}
$$

where $\langle\dot{\gamma}\rangle_{s}$ is the bulk shear rate at the interface, $\langle u\rangle_{s}$ is the interfacial slip velocity, and $\lambda$ is the slip length. For superhydrophobic surfaces, the dependence of $\lambda$ on the geometry of the microstructure has been studied extensively (Rothstein 2010): $\lambda$ is a function of the size of the microstructural elements, the area fraction covered by the solid, and the arrangement of the microstructural elements in the slip plane (Ybert et al. 2007; Sbragaglia \& Prosperetti 2007; Lauga \& Stone 2003; Ng \& Wang 2009). In this paper we investigate the suitability of boundary condition (1.1) forl flat particle-laden gas-liquid interfaces; results for flat interfaces are relevant to flow situations in which the radius of curvature of the interface is much larger than the characteristic particle radius. We characterise the slip flow past a monolayer of stationary spherical particles for a specific contact angle, $\theta_{c}=90^{\circ}$, and investigate the dependence of $\lambda$ on relevant parameters for two cases: a square array, and a reticulated array in which the particles are distributed according to a mesh-like arrangement. These two cases are idealisations of two limiting cases found in practice (Aveyard et al. 2000) of monolayers constituted by particles well dispersed in the interface owing to interparticle repulsion, and monolayers constituted by particles forming two-dimensional percolating networks owing to particle-particle attraction, respectively.

The neutrally-wetting case $\theta_{c}=90^{\circ}$ studied here has practical relevance. In applications it is indeed desirable to have a contact angle close to $90^{\circ}$, as this limiting angle gives the strongest adhesion of the particle to the interface against desorption in either of the two adjacent fluids (Binks \& Horozov 2006).

\section{Problem formulation}

We model shear flow past a stationary monolayer of spherical particles of radius $a$ embedded in a flat air-liquid interface for a contact angle of $90^{\circ}$ (figure 2). The fluid is set in motion by a flat wall located at a distance $d$ from the monolayer and translating with velocity $U$ with respect to the spheres. For large values of $d$ the liquid can be considered to be bounded by the particle-laden interface only. In this limit we obtain asymptotic results that depend on the macroscopic bulk shear rate, and not on $d$ directly.

To simulate the flow configuration described above, we employ an expedient that allows 


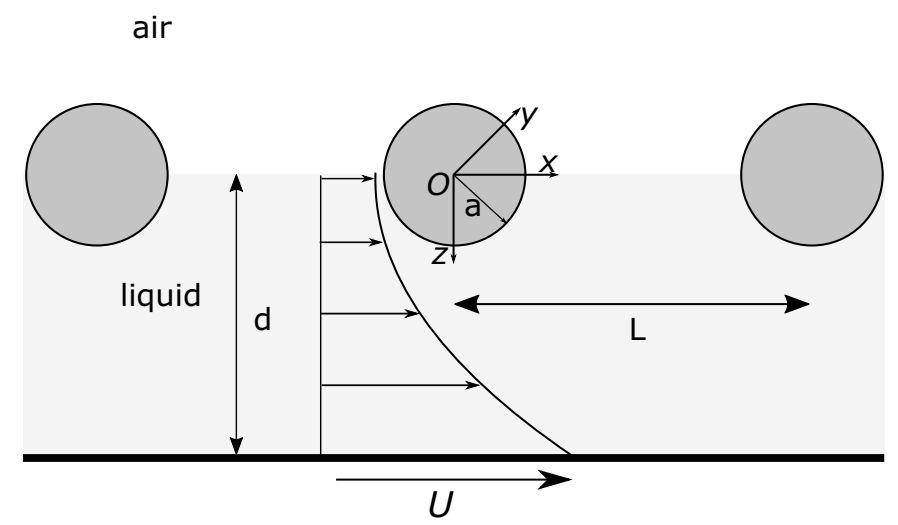

Figure 2: We simulate a stationary monolayer of particles half-immersed in a flat gasliquid interface. The fluid is sheared by a moving wall located at a distance $d$ from the monolayer and moving with constant velocity $U$. For large values of $d$ our results converge to the asymptotic limit in which the flow near the monolayer depends on the macroscopic shear rate induced by the moving wall, but not on $d$ directly.

to use a fast solver for finite-size particles in bulk flows to simulate a particle-laden gasliquid interface. We simulate the flow past a monolayer of spherical particles completely embedded in the liquid and placed at the center of a two-dimensional channel. The channel walls translate parallel to the monolayer with velocity $U$. Because the air-water free-shear interface is a plane of symmetry for the flow, the flow below the monolayer with the fully-immersed particles is identical to the flow in which the particles are halfimmersed in a liquid domain bounded from the top by the air-water interface (Dörr \& Hardt 2015). The use of this expedient enables us to use a fast and accurate fixed-grid method for fully-resolved particles, Physalis (Zhang \& Prosperetti 2005; Sierakowski 2016), to simulate at a reasonable computational cost many particles embedded in a gas-liquid interface.

Following recent work on particles at interfaces (Dörr \& Hardt 2015; Dörr et al. 2016), we neglect the rotation of the particles. This assumption holds when the viscous dissipation due to the rotation of the particles is negligible in comparison to the dissipation due to the particle-fluid velocity difference (Dörr et al. 2016) or when the motion of the contact line is hindered due to pinning of the contact line at roughness elements or chemical heterogeneities (Dörr \& Hardt 2015; Dörr et al. 2016). The maximum pinning force per unit length of contact line is approximately equal to $\sigma \Delta$, where $\sigma$ is the surface tension of the air-liquid interface and $\Delta$ is the difference between the cosines of the advancing and receding contact angles (De Gennes 1985). The parameter $\Delta$ is often not negligible (the difference between the advancing and receding contact angles is often larger than $10^{\circ}$, see e.g. Lewandowski et al. (2010)) and this translates to finite torque due to pinning that scales as $\sigma a^{2}$. The hydrodynamic torque on each particle is $O\left(\mu \dot{\gamma} a^{3}\right)$, where $\mu$ is the liquid viscosity and $\dot{\gamma}$ is the characteristic value of the shear rate near the particle monolayer. The ratio of the torque due to pinning to the hydrodynamic torque is thus proportional to the capillary number $C a=\mu \dot{\gamma} a / \sigma$. Our results are valid in the limit 
$C a \ll 1$, in which the particle does not rotate and hydrodynamic stresses are too small to appreciably deform the interface. Small capillary numbers are often found in practice: for example for a particle-covered bubble of radius $R=100 \mu \mathrm{m}$ translating with a velocity $U_{\infty}=1 \mathrm{~cm} / \mathrm{s}, \dot{\gamma} \sim U_{\infty} / R=100 \mathrm{~s}^{-1}$ and $C a \sim 10^{-6}$. The capillary number for the entire drop based on the velocity scale $U_{\infty}$ is a factor $R / a$ larger than $C a$ (which is based on the velocity scale $\dot{\gamma} a$ ), but is still very small.

We consider plane monolayers of particles arrangements in a bi-periodic configuration for two situations: a square array, and a reticulated mesh-like array formed by orthogonal chains of particles with one of the chains oriented parallel to the flow.

The problem is governed by two non-dimensional parameters: the non-dimensional gap size $d / a$ and the solid area fraction $\phi_{s}=\frac{N_{p} \pi a^{2}}{L^{2}}$, where $N_{p}$ is the number of particles in the periodic cell of side $L$. For the simulation of the square array, we simulate a single sphere and vary the area fraction by changing the lateral size of the computational domain. For the reticulated array case, examined in section $3.4, N_{p}$ varies from 5 to 25.

A Cartesian coordinate system $(x, y, z)$ is set at the centre of the computational domain, with $x$ parallel to the flow direction and $z$ in the direction normal to the monolayer. In the following, $u$ will denote the flow velocity component in the $x$ direction.

The numerical method employed for the simulation, Physalis, couples a finite-difference solution of the incompressible Navier-Stokes equation to a spectral solution of the Stokes equation for the velocity, vorticity and pressure disturbances induced by the sphere. The spectral solution is used only in the immediate neighborhood of the particle surfaces. The pressure and vorticity are expressed in terms of spherical harmonics. To enforce the no-slip condition at the particle surfaces, an iterative procedure is used to match the coefficients of the spherical harmonics expansion to the finite-difference Navier-Stokes solution at a cage of computational points surrounding the particle surface. For the simulations in the current paper, we use the Navier-Stokes solver with the non-linear convective term set to zero (i.e., all the simulations are carried out in the Stokes flow limit). The results we report are for steady-state conditions. Physalis has been extensively validated in laminar flows (Zhang \& Prosperetti 2005; Bluemink et al. 2008, 2010). It has been applied to a shear flow over a porous medium surface composed of several layers of spheres (Liu \& Prosperetti 2011), a situation that bears some similarities with the flow simulated in the current work.

The accuracy of the simulation was assessed through comparison with power-series Faxén's analytical solution for the drag force on a single sphere translating with constant velocity between two parallel walls (Happel \& Brenner 2012). Terms up to $O\left(d^{5} / a^{5}\right)$ were retained in the power series. Our numerical results for the drag force on a fullyimmersed sphere in a periodic domain converged to Faxén's solution in the limit $\phi_{s} \rightarrow 0$. The relative error between the numerical solution - for small but finite values of $\phi_{s}$ and the analytical solution was found to be always less then $5 \%$, and typically around $2.5 \%$. For example, for $\phi_{s}=0.349 \%$ the relative error between the numerical solution and Faxén's solution is $3.89 \%$ and $2.57 \%$ for $d / a=3$ and $d / a=15$, respectively.

Owing to the use of a spectral solution close to the particle surface, the Physalis method demonstrates good accuracy even in simulations in which a relatively small number of nodes per particle diameter is used (Zhang \& Prosperetti 2005; Bluemink et al. 2008; Botto \& Prosperetti 2012). For the simulations in this paper we used either 8 or 16 nodes per particle diameter. The smaller resolution was used for relatively large gaps, $d / a>5$, and small area fractions, $\phi_{s}<0.15$, for which the flow velocity gradients are small. These values of the parameters correspond to large domains for which computational cost and memory requirements were found to be limiting factors. The simulations are carried 
out on a desktop PC equipped with an NVIDIA GTX 970 graphic card (the version of Physalis used here, BlueBottle, has the ability to exploit the GPU card of the PC).

Unless explicitly stated, all the quantities reported in this paper are normalised using the particle size $a$ and the wall velocity $U$ as the characteristic length and velocity scales, respectively.

\section{Results and discussion}

\subsection{Square array: general features}

We begin our analysis by examining the square array case. The periodic computational domain of side $L$ contains in this case a single sphere, and the area fraction can be simply calculated as $\phi_{s}=\pi a^{2} / L^{2}$. We are particularly interested in the area averaged streanwise velocity $\langle u\rangle$, defined as

$$
\langle u\rangle=\frac{1}{L^{2}} \int u d x d y
$$

and how this quantity changes in the direction normal to the monolayer. In equation 3.1 the integral is extended over the region $-L / 2 \leq x \leq L / 2,-L / 2 \leq y \leq L / 2$.

In figure $3 \mathrm{a}$, the area averaged velocity is plotted as a function of the coordinate $z$ normal to the monolayer for a fixed gap size, $d=3 a$. The velocity profile is approximately linear in two limits: when $\phi_{s} \ll 1$ and when $\phi_{s}$ is close to the maximum packing fraction $\phi_{s, \max }=\pi / 4 \simeq 0.78$. For intermediate values of $\phi_{s}$, the curvature of the velocity profile has a maximum. This trend can be understood by applying the averaging operator defined in (3.1) to the streamwise component of the fluid momentum equation in the Stokes flow limit. The resulting averaged equation reads

$$
\frac{d^{2}\langle u\rangle}{d z^{2}}=\frac{\Delta \bar{p}}{\mu L},
$$

where $\Delta \bar{p}(z)$ is the difference between the pressure at the plane $x=-L / 2$, averaged over the line $-L / 2 \leq y \leq L / 2$, and the corresponding average pressure at the plane $x=L / 2$. Expression 3.2 shows that the curvature of the average velocity profile can be neglected when the streamwise pressure drop occurring over a distance $L$ is negligible.

According to the Stokes equation, the pressure disturbance induced by the particle determines the curvature of the velocity profile. When $\phi_{s} \ll 1, \Delta \bar{p} \simeq 0$ because the pressure disturbance set up by each sphere evaluated at the boundaries of the computational domain is small. Indeed, we will shortly see that in the dilute limit the pressure disturbance induced by a particle looks like a pressure dipole, and in the dilute limit we therefore expect $\Delta \bar{p}=O\left(\mu a U / L^{2}\right)$ (Batchelor 2000), as for a single sphere in uniform flow. The pressure disturbance $\Delta \bar{p}$ decreases linearly with $\phi_{s}$ as $\phi_{s} \rightarrow 0$, since $\phi_{s} \propto 1 / L^{2}$. On the other hand, when the monolayer is near maximum packing the monolayer behaves almost as a flat wall. In this case, $\Delta \bar{p} \simeq 0$. For intermediate values of $\phi_{s}$, the inter-particle distance is simultaneously sufficiently small for the pressure disturbance produced by each particle on the boundaries of the computational domain to be significant, and sufficiently large for each particle not to block significantly the flow velocity incident on the other particles. As a consequence, the curvature has a maximum for intermediate values of $\phi_{s}$.

To characterise how the velocity profile changes in the plane of the monolayer, we show in figure $3 \mathrm{~b}$, top and bottom panels, profiles of $u$ along lines perpendicular to the plane of the monolayer and passing through the sphere centre, $(x=0, y=0)$, and through the midpoint between two spheres, $(x=L / 2, y=0)$, respectively. Because of the fore-aft 


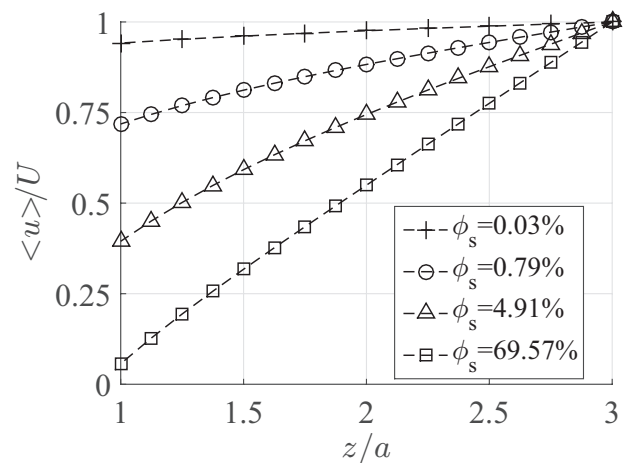

(a)
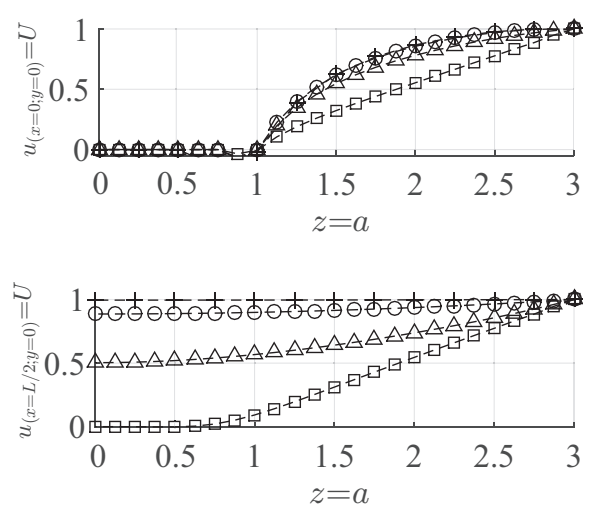

(b)

Figure 3: (a) Normalised area averaged streamwise velocity vs. coordinate normal to the monolayer for $d=3 a$. (b) Normalised area averaged streamwise velocity along lines passing through the centre of each sphere (top figure) or through the midpoint between adjacent spheres (bottom figure).

symmetry of the Stokes equation, the velocity disturbances generated by two adjacent spheres cancel out at the midpoint $(x=L / 2, y=0)$. As a consequence, the velocity profiles corresponding to the midpoint are linear for any value of $\phi_{s}$. As $\phi_{s}$ is reduced, the slope of the velocity profiles decreases. This trend gives a larger slip velocity. The velocity profiles that correspond to the sphere centres (figure $3 \mathrm{~b}$, bottom panel) display significant curvature for any $\phi_{s}$, except perhaps at the highest value of the area fraction.

The error induced by approximating a liquid interfaces covered by a packed monolayer of particles as a no-slip surface is due to two sources. First of all, a packed monolayer contains free-slip surfaces even at maximum packing. Secondly, due to the convexity of the spheres the packed monolayer is not flat. A more accurate interpretation is considering the monolayer as a collection of bluff solid protuberances over the no-shear plane $z=0$. Each protuberance will locally block the flow and therefore produce a significant pressure disturbance.

To illustrate the spatial extent and magnitude of the pressure disturbance set up by each sphere in the monolayer, we show in figure $4 \mathrm{a}$ and $4 \mathrm{c}$ iso-contours of the normalised pressure in the plane parallel to the mean flow and perpendicular to the monolayer, for two selected values of the area fraction, $\phi_{s}=12.57 \%$ and $\phi_{s}=62.05 \%$. For $\phi_{s}=12.57 \%$, the pressure distribution bears signature of the fore-aft symmetric pressure dipole characteristic of uniform flow past a sphere (Batchelor 2000). As the area fraction increases (Figure 4b), the two pressure "lobes" seen in 4a move upwards, and occupy a smaller region near the top apex of the sphere. The streamwise pressure drop caused by the sphere is smaller for $\phi_{s}=62.05 \%$ than for $\phi_{s}=12.57 \%$. This observation supports our suggestion that $\Delta \bar{p}$ decreases as $\phi_{s}$ approaches the maximum packing fraction.

Figure $4 \mathrm{~b}$ and $4 \mathrm{~d}$ show the velocity fields corresponding to figures $4 \mathrm{a}$ and $4 \mathrm{c}$, respectively. Flow recirculation regions do not seem to occur between the spheres. Flow recirculation between the spheres were evidenced in a simulation of pressure-driven slip flow over a porous medium interface, where the simulations were carried out using the same numerical method used here (Liu \& Prosperetti 2011). Flow recirculation is instead not apparent in the simulation results reported by Danov et al. (1995), who examined a 


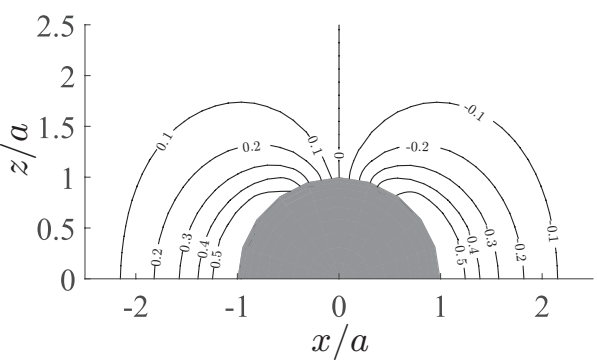

(a)

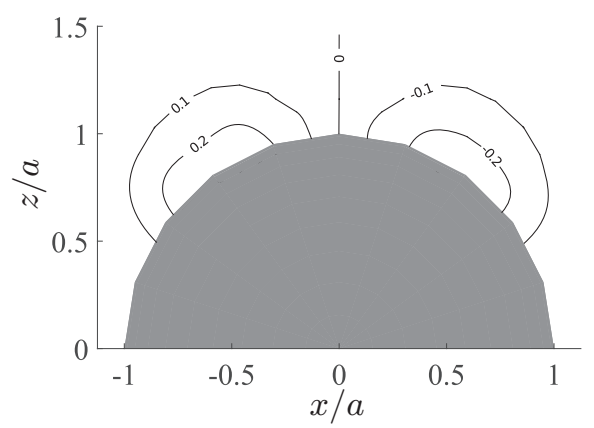

(c)

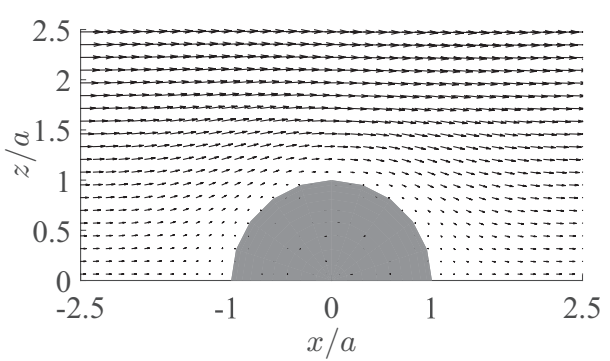

(b)

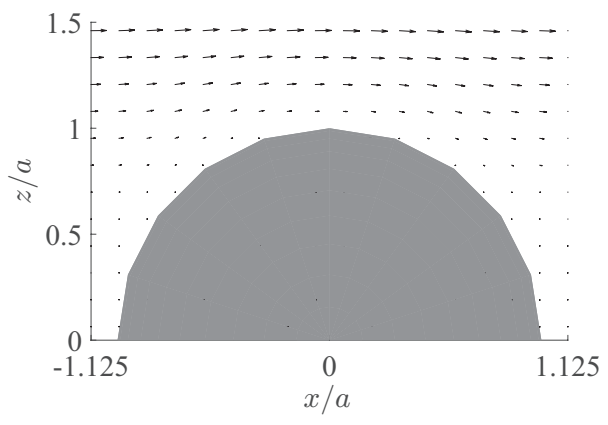

(d)

Figure 4: Normalised pressure (left) and velocity (right) in the plane $y=0$ for $\phi_{s}=12.57 \%$ (top panels) and $\phi_{s}=62.05 \%$ (bottom panels). The gap size is $d=4 a$.

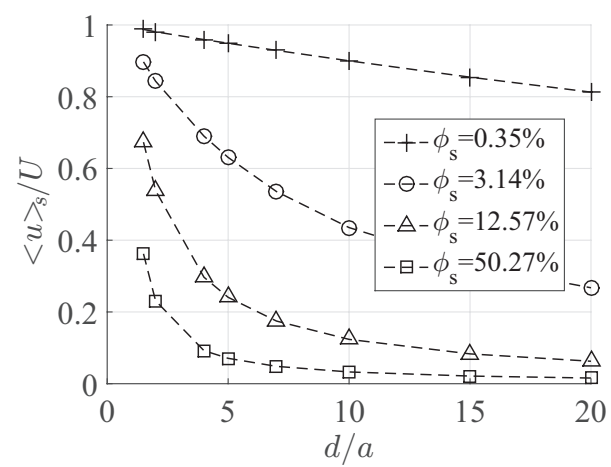

(a)

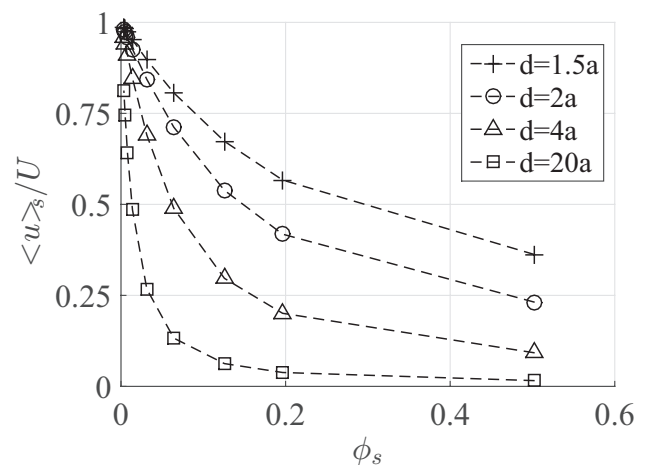

(b)

Figure 5: Normalised slip velocity vs. (a) area fraction and (b) gap size.

single sphere straddling a fluid interface for a range of contact angles. One could expect that, owing to the presence of a re-entrant "wedge region" between the particle surface and the free interface, recirculation would occur for contact angles for which the sphere is mostly immersed in the liquid.

The slip velocity is plotted as a function of $\phi_{s}$ for different values of the gap size $d$ in figure $5 \mathrm{a}$, and as a function of $d$ for different values of $\phi_{s}$ in figure 5b. Following other authors (Ng \& Wang 2009; Ybert et al. 2007; Liu \& Prosperetti 2011), we define the slip 
velocity $\langle u\rangle_{s}$ as the value of $\langle u\rangle$ at $z=a$. As expected $\langle u\rangle$ tends to a uniform velocity of magnitude $U$ as $\phi_{s} \rightarrow 0$. The rate at which this limit is approached as $\phi_{s}$ changes depends on the gap size. As a consequence, the value $\phi_{s}$ for which $\langle u\rangle$ is a significant fraction of $U$ becomes smaller as $d$ increases. For instance, when $d / a=20,\langle u\rangle / U \simeq 0.5$ when $\phi_{s} \simeq 1.5 \%$. When $d / a=1.5,\langle u\rangle / U \simeq 0.5$ when $\phi_{s} \simeq 30 \%$. Since smaller gaps are associated with larger velocity gradients, this dependence on $d$ indicates a dependence of $\langle u\rangle_{s}$ on the shear rate.

The dependence of $\langle u\rangle_{s}$ on $d$ is non-linear, but approximates a linear relation in the limit $\phi_{s} \rightarrow 0$ (figure $5 \mathrm{~b}$ ). The following argument shows that in the dilute limit the slope of the $\langle u\rangle_{s}$ vs. $d$ curve is proportional to the gap size and inversely proportional to the slip length. Approximating the flow past the monolayer as a Couette flow past a flat partial slip surface, the macroscopic shear rate is $\langle\dot{\gamma}\rangle_{s} \simeq\left(U-\langle u\rangle_{s}\right) / d$. Using this value in the Navier slip boundary condition (1.1) gives $\langle u\rangle_{s} / U \simeq \frac{1}{d / \lambda+1}$. For $\phi_{s} \ll 1, \lambda$ becomes much larger than $d$ and therefore $\langle u\rangle_{s} / U=1-\frac{d}{\lambda}$ with a small $O(d / \lambda)$ error.

\subsection{Scaling of slip length for square array}

Figure 6 shows the slip length $\lambda$ as calculated from the definition (1.1). Consistently with the the calculation of the slip velocity, the bulk shear rate at the interface $\langle\dot{\gamma}\rangle_{s}=d\langle u\rangle / d z$ is evaluated at $z=a$. In the surface plot of 6 a, $\lambda$ is plotted as a function of both $\phi_{s}$ and $d / a$. Projections of figure 6 onto the $\lambda-d$ and $\lambda-\phi_{s}$ planes are shown in linear-linear plots in figures $6 \mathrm{~b}$ and $\mathrm{c}$, respectively. The inset of figure $6 \mathrm{c}$ shows the $\lambda-\phi_{s}$ relation in $\log -\log$ scale.

The slip length is seen to increase for increasing gap sizes, eventually saturating to an asymptotic value. A gap size $d=10 a$ already gives a value of $\lambda$ close to the asymptotic limit.

In figure $6 \mathrm{c}$, the values of $\lambda$ for different values of $d$ are seen to lay practically on the same curve, suggesting similar scaling laws for different gap sizes. The inset to this figure shows that the relation $\lambda-\phi_{s}$ follows approximately a power law. To guess possible scaling exponents, we have examine the literature on flows over microstructured super-hydrophobic surfaces. Ybert et al. (2007) developed a comprehensive theory for the dependence of the slip length on the area fraction for super-hydrophobic surfaces. The theory was compared against literature data. Data for unconfined shear flow past a superhydrophobic surface composed of vertical pillars of circular or square cross section could be fitted with good accuracy by a correlation of the form $\frac{\lambda}{L}=\frac{A_{1}}{\sqrt{\phi_{s}}}-B_{1}$, where $L$ is the distance between the pillars, and $A_{1}$ and $B_{1}$ are constant. Ybert et al. (2007) proposed $A_{1}=0.325$ and $B_{1}=0.44$ (the analytical expressions proposed by Davis \& Lauga (2010) give coefficient values very close to those indicated by Ybert et al. (2007)). In the dilute limit, i.e. in our case for $\phi_{s} \ll\left(A_{1} / B_{1}\right)^{2} \simeq 0.54$, the correlation above reduces to $\lambda / L \sim 1 / \sqrt{\phi_{s}}$, which is equivalent to $\lambda / a \sim 1 / \phi_{s}$. This scaling can be understood from the fact that in the dilute limit the ratio of the hydrodynamic force on each pillar to the slip velocity is expected to be practically independent of $\phi_{s}$. The tangential stress on the monolayer due to the bulk flow is proportional to the ratio of the hydrodynamic force and $L^{2}$. Since the tangential stress for a Newtonian fluid is proportional to the macroscopic shear rate, then Eq. (1.1) yields $\lambda \propto a / \phi_{s}$ for $\phi_{s} \ll 1$.

The argument above is expected to hold independently of the specific geometry of the solid object in contact with the fluid interface. Therefore, it should be possible to apply the scaling proposed by Ybert et al. (2007) to our case. This expectation is confirmed in figure $7 \mathrm{a}$, where $\lambda / a$ is plotted as a function of $1 / \phi_{s}$ for values of $\phi_{s}$ corresponding to a relatively dilute monolayer. A linear correlation fits the data remarkably well. From 


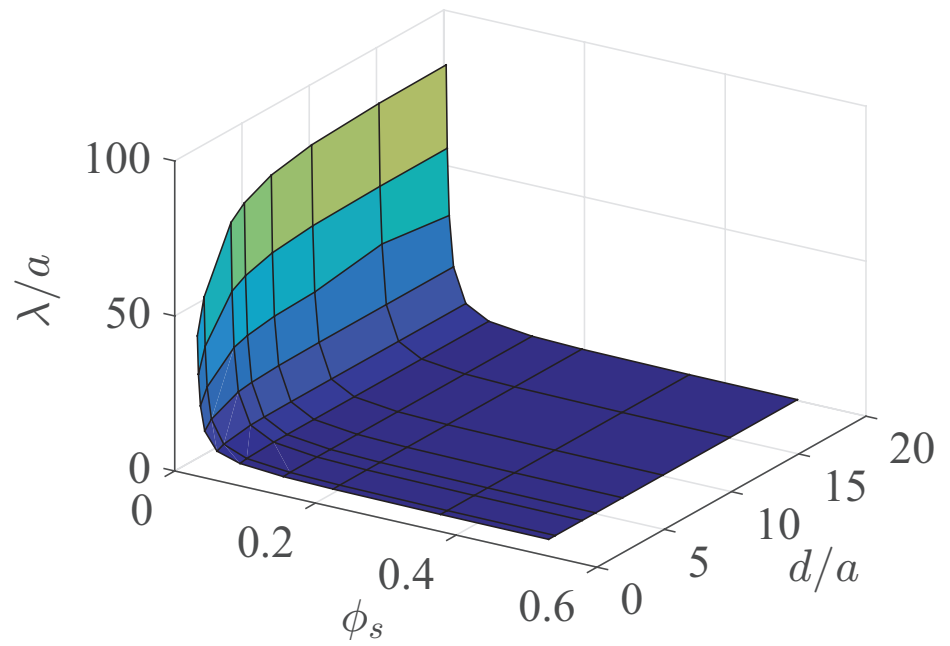

(a)

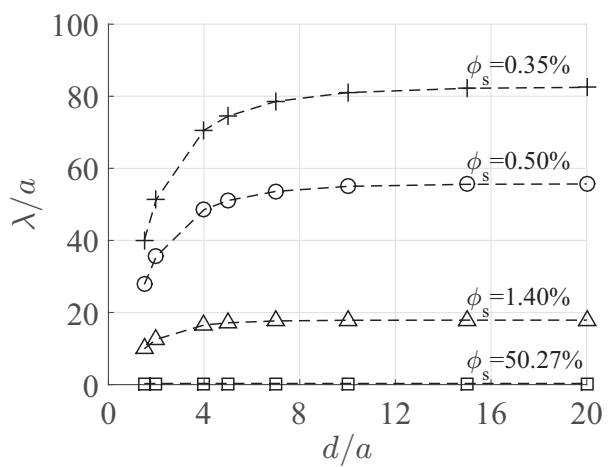

(b)

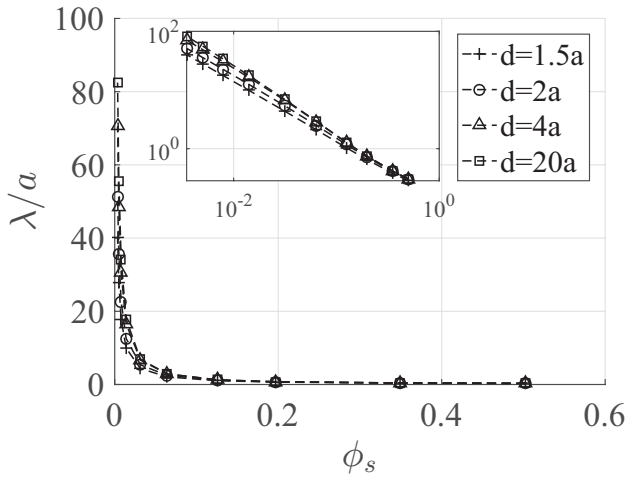

(c)

Figure 6: (a) Normalised slip length vs. area fraction and normalised gap size. The plots in figures (b) and (c) are projections of the surface plot of figure (a) onto the $\lambda-d$ and $\lambda-\phi_{s}$ planes, respectively. Inset: $\lambda-\phi_{s}$ relationship in $\log -\log$ scale.

figure 7a it appears that a linear scaling holds for any value of $d$. The log-log plot in the inset of figure $6 \mathrm{~b}$ shows that the power law exponent has a small dependency on $d$ for $d \leq 4 a$. However, differences between the exponents corresponding to different values of $d$ are about $10 \%$ of the $d=20 a$ case, and therefore not noticeable if the data is plotted as in figure $7 \mathrm{a}$.

While the functional form proposed by Ybert and collaborators does fit our data well, the prefactors are different. The function $\frac{\lambda}{a}=\frac{A_{1} \sqrt{\pi}}{\phi_{s}}-\frac{B_{1} \sqrt{\pi}}{\left(\phi_{s}\right)^{1 / 2}}$, obtained using the definition $\phi_{s}=\pi a^{2} / L^{2}$ is plotted as a continuous line in figure $7 \mathrm{a}$, using the coefficient values for $A_{1}$ and $B_{1}$ suggested by Ybert $e$ t al. This function is seen to overpredict the magnitude of the slip length computed in our simulation.

There is a simple explanation for the difference between our result and that of Ybert et al.The flow configuration considered by these authors can be interpreted as shear flow past a monolayer of infinitely thin disks (representing the top surfaces of the pillars 


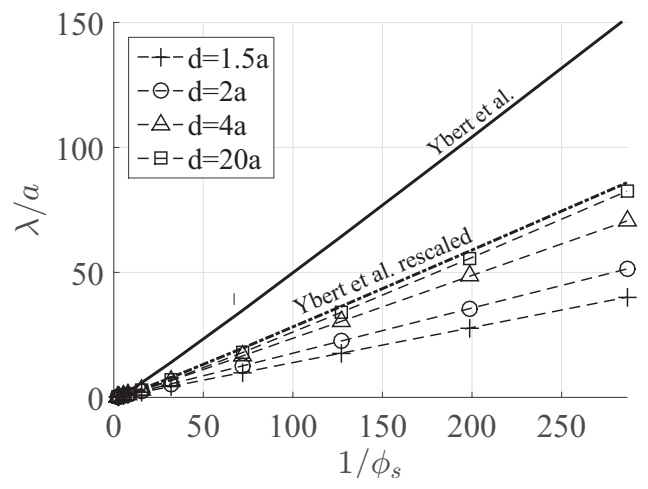

(a)

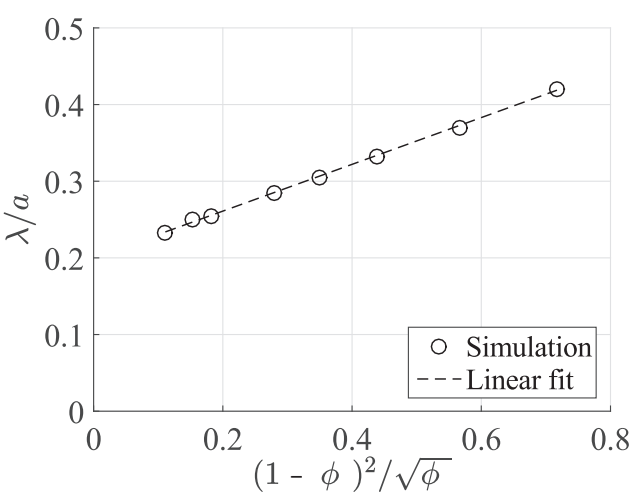

(b)

Figure 7: (a) Normalised slip length vs. $\phi_{s}^{-1}$. The continuous line is equation (3.2), developed by Ybert et al. (2007) for flow past a superhydrophobic surface. The dashed line is equation (3.3), where (3.2) has been rescaled by the ratio of the Stokes drag coefficient for a sphere to that for a thin disk. (b) Normalised slip length vs. $\frac{\left(1-\phi_{s}\right)^{2}}{\sqrt{\phi_{s}}}$ showing the dense limit $\phi_{s}=35 \%-70 \%$ for $d=4 a$.

composing the super-hydrophobic surface). In our case the spheres protrude significantly in the liquid. Several studies in the context of super-hydrophobic surfaces have shown that a larger protrusion produces smaller slip lengths (Sbragaglia \& Prosperetti 2007; Ng \& Wang 2009; Kumar et al. 2016; Shelley et al. 2016). Therefore a smaller slip length in the current case of sphere monolayer is not unexpected.

We can attempt a simple correction to equation (3.2) to account for the finite protrusion of the spheres into the flow. For a given free-stream velocity the hydrodynamic drag on an infinitely thin disk in a uniform flow is $9 \pi / 16$ times smaller than the drag on a sphere having the same radius. Assuming that tangential stress due to the bulk flow is approximately proportional to the slip velocity (we will confirm this hypothesis in Sec. 3.3 ), it should be expected that for sufficiently dilute systems the slip length for flow past a monolayer of spheres embedded in a gas-liquid interface is a factor of $9 \pi / 16$ smaller than that predicted by equation 3.2. To verify this approximation we plot the function

$$
\frac{\lambda}{a}=\frac{A_{2}}{\phi_{s}}-\frac{B_{2}}{\left(\phi_{s}\right)^{1 / 2}},
$$

as a thick dashed line in figure 7 a. Here $A_{2}=\frac{16 \pi \sqrt{\pi}}{9} A_{1}$ and $B_{2}=\frac{16 \pi \sqrt{\pi}}{9} B_{1}$. The values given by this corrected expression are remarkably close to our data, suggesting that the difference between the data of Ybert et al. and ours can be mainly attributed to the larger drag produced by the protruding spheres on the flowing liquid as opposed to the flat top surfaces of the pillars.

The scaling law discussed above holds for a relatively dilute limit (the data points clearly discernible in figure 7 a correspond to $\phi_{s} \leq 4 \%$, while these corresponding to the dense limits are clustered near the origin and are barely visible). In the dense limit, the slip velocity results from a fraction $1-\phi_{s}$ of the plane $z=a$ occupied by the gas-liquid interfaces. Since the slip velocity in these regions is of the order of $\langle\dot{\gamma}\rangle_{s} L\left(1-\phi_{s}\right.$ ) (Ybert et al. 2007) and $L \propto \phi_{s}^{-1 / 2}$, we expect $\lambda \sim a \frac{\left(1-\phi_{s}\right)^{2}}{\sqrt{\phi_{s}}}$. Figure $7 \mathrm{~b}$ shows that the simulation data follows this scaling law with very good accuracy. 


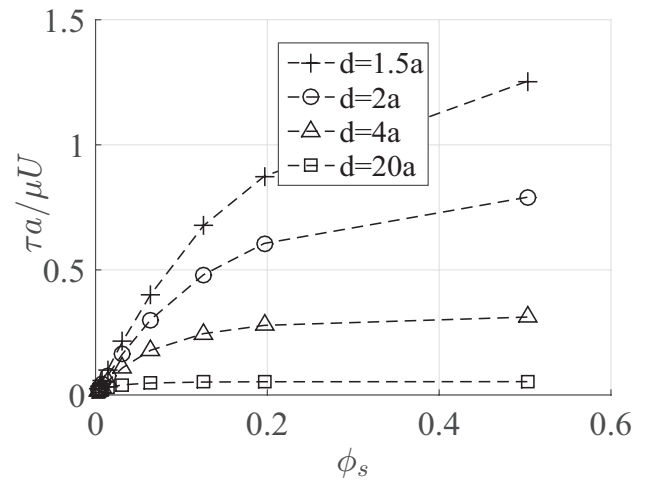

(a)

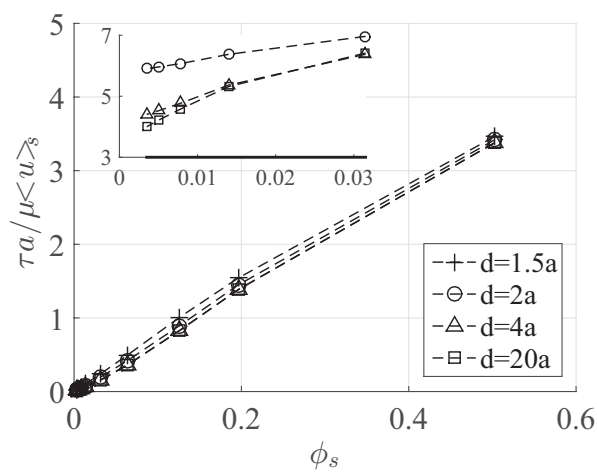

(b)

Figure 8: Tangential stress due to the bulk flow normalised by (a) the wall velocity $U$ or (b) the slip velocity $\langle u\rangle_{s}$ as a function of the solid area fraction. The inset in figure (b) shows a zoom for small values of $\phi_{s}$ of the effective friction coefficient $\tau a /\left(\mu\langle u\rangle_{s} \phi_{s}\right)$.

\subsection{Tangential stress due to the bulk flow: square array}

In many situations of practical interest it would be useful to estimate the drag per unit area on the particles due to the motion of the bulk fluid (or "subphase', as often called in the surfactants literature). We call the hydrodynamic drag on the particles per unit area the tangential stress due to the bulk flow, and denote this quantity by $\tau$. In the current paper, $\tau$ for a square array is calculated as $\tau=F_{x} /\left(L^{2}\right)$, where $F_{x}$ is the $\mathrm{x}$-component of the hydrodynamic force acting on each sphere in the monolayer.

In figure 8a, $\tau$ is normalised by using $U$ as velocity scale. With this normalisation the normalised value of $\tau$ has a relatively strong dependence on both $\phi_{s}$ and $d$. However, we note that the wall velocity is characteristic of the fluid velocity "seen" by the particles only in the extremely dilute limit for which the velocity profile is almost a plug flow. An improved parameterisation of the shear stress and pressure exerted on each particle by the bulk flow is expected to be given by the slip velocity $\langle u\rangle_{s}$. In figure $8(\mathrm{~b}), \tau$ is normalised by $\langle u\rangle_{s}$. Using the slip velocity in the normalisation gives a collapse of the data onto a single curve for any value of $d$.

The fact that $\tau$ is practically proportional to the slip velocity suggests to introduce a non-dimensional friction coefficient $\tau a /\left(\mu\langle u\rangle_{s} \phi_{s}\right)$ having only a marginal dependence on $d$ and $\phi_{s}$. A linear fit to the data in figure 8 (b) suggests a non-dimensional friction coefficient of about 7 . For small values of $\phi_{s}$ the friction coefficient is smaller than 7 and non constant (see inset), suggesting that the relation between $\tau /\langle u\rangle_{s}$ and $\phi_{s}$ is linear only in an approximate sense.

In the limit $\phi_{s} \rightarrow 0$ and $d \rightarrow \infty$ we expect $F_{x} \simeq 3 \pi \mu a U$ (i.e. half the Stokes drag on a fully immersed sphere). Since $\langle u\rangle_{s}$ tends to $U$ as $\phi_{s} \rightarrow 0$ (Fig.5a), and by definition $\tau=F / L^{2}=F \phi_{s} /\left(\pi a^{2}\right)$ for a square array, one might expect a friction coefficient of approximately 3 in the limits $\phi_{s} \rightarrow 0$ and $d \rightarrow \infty$. For small values of $\phi_{s}$ the data does appear to converge to a friction coefficient of 3 as $d$ increases (see inset of figure $8 \mathrm{~b}$ ). However, the convergence is slow and for values we simulated the friction coefficient is significantly larger than 3 even for the smallest values of $\phi_{s}$ we considered. This fact may be due to the strong dependence of $\langle u\rangle_{s}$ on the surface fraction (Fig.5a). One would need to simulate truly negligible values of $\phi_{s}$, and use therefore extremely large computational domains, to recover the expected asymptotic limit. 


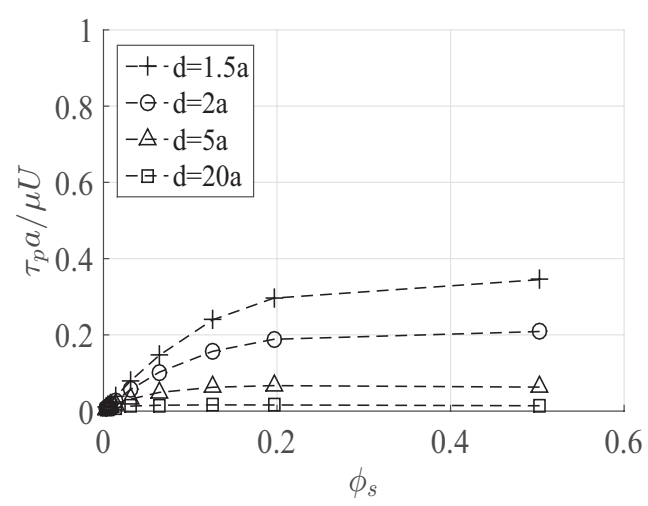

(a)

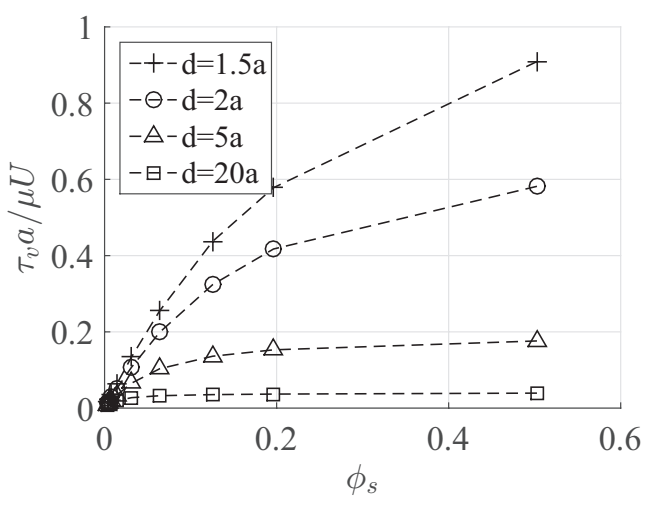

(b)

Figure 9: Normalised pressure (a) and viscous (b) contributions to $\tau$.

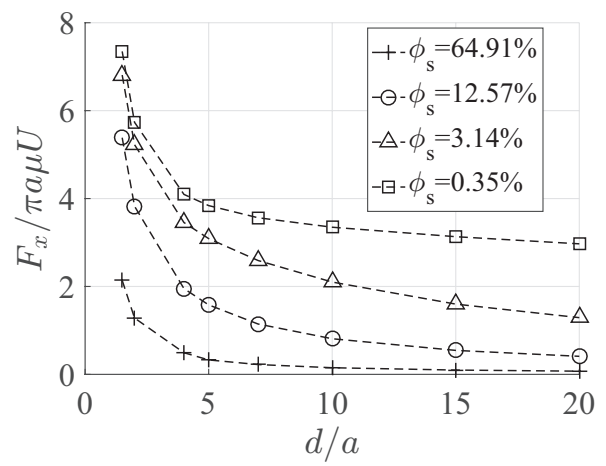

Figure 10: Hydrodynamic force on each sphere in the square array as a function of the gap size.

The expansion in spherical harmonics in Physalis enables to easily compute the pressure and viscous components of the hydrodynamic force on each sphere with excellent accuracy (Zhang \& Prosperetti 2005; Bluemink et al. 2010; Botto \& Prosperetti 2012). Upon normalisation, these force components yield $\tau_{p}$ and $\tau_{v}$, namely the pressure and viscous contributions to $\tau$, respectively. Figures 9 (a) and (b) show $\tau_{p}$ and $\tau_{v}$ as a function of $\phi_{s}$ for different values of $d$. As expected from the analytical solution for Stokes flow past a sphere, which suggest that pressure and viscous contributions to the drag are comparable, for $\phi_{s} \ll 1$ the viscous and pressure contributions to $\tau$ are comparable in magnitude. For intermediate and relatively large values of $\phi_{s}$, viscous stresses produce the dominant contribution to $\tau$. For instance, for $d=7 a$ and $\phi_{s}>0.5, \tau_{v}$ is about one order of magnitude larger than $\tau_{p}$. The contribution $\tau_{p}$ is only significant for small gap sizes, particularly at the highest values of the area fraction. This result confirms the intuitive notion that the largest contribution to $\tau$ for a moderately dense monolayer originates from the shear forces exerted by the fluid on the portion of the sphere surfaces where the streamwise fluid velocity is larger.

For completeness, we also report in figure 10 the hydrodynamic force on each particle in the square monolayer as a function of $d$ for different values of $\phi_{s}$. In contrast to the previous figures in which the tangential stress due to the bulk flow was reported, in figure 


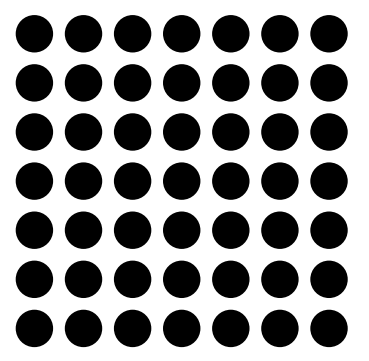

(a)

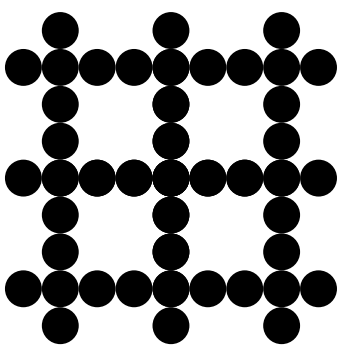

(b)

Figure 11: Schematic of a square array (a) and a reticulated array (b) for the same value of the solid area fraction, $\phi_{s}=43.63 \%$.

10 the force is not normalised by $L^{2}$, and therefore the dependence on $\phi_{s}$ is perhaps more clear. This graph could be useful to quantify how the drag on a surface active particle embedded at the boundary of a thin film comprised between a particle-laden interface and a wall changes as a function of the thickness of the film.

\subsection{Reticulated array}

The slip length depends on the specific microstructure of the monolayer. Two categories of microstructures are particularly relevant to particle-laden interfaces: well-dispersed systems - in which the particles are not in contact when $\phi_{s}$ is smaller than the maximum packing fraction - and percolating networks, where the particles form connected chains that span the boundaries of the interface (see e.g. Aveyard et al. (2000)). The squarearray case examined in the previous section belongs to the first category. To percolating network case is examined in the current section. To get initial insights into more realistic situations, in which the percolating networks are disordered and are characterised by a variety of scales, in this section we simulate a periodic mesh-like reticulated arrangement. One example of such arrangement is illustrated in figure $11 \mathrm{~b}$.

The simulations for the reticulated array case are carried out by including in the doubly-periodic simulation box a variable number of spheres. The spheres form two orthogonal rectilinear chains that intersect each other in the center of the domain. One of the chains is oriented along the flow direction.

Because of the need to consider several particles, simulating reticulated arrays requires a significantly larger domain than for a square array, for a given area fraction. To limit the number of simulations while allowing to explore a relevant parameter space, the majority of the simulations presented in this section are carried out for a fixed gap size, $d=7 a$. Preliminary tests confirm that this gap size is sufficiently large for the results to reasonably approximate the unbounded case $d=\infty$.

Table 1 summarises values of the slip length $\lambda$ and tangential stress $\tau$ due to the bulk flow for different values of $L$ (and therefore of $\phi_{s}$ ). The last two columns in table 1 report the values of $\lambda$ and $\tau$ for a square array having the same area fraction of the corresponding reticulated array. These two quantities are denoted by the symbols $\lambda_{s q}$ and $\tau_{s q}$, respectively.

Comparison of $\lambda$ with $\lambda_{s q}$ shows that for a given surface coverage and particle size, the reticulated array gives a significantly larger value of the slip length. The effect of the specific microstructure (square array vs. reticulated array) on $\lambda$ becomes more and more significant as $\phi_{s}$ decreases. The difference between $\tau$ and $\tau_{s q}$ is comparatively small.

Why does the reticulate array give a larger slip length?The slip length is defined as 


\begin{tabular}{ccccccc}
\hline Structure & $\phi_{s}$ & $L / a$ & $\lambda / a$ & $\tau a / \mu U$ & $\lambda_{s q} / a$ & $\tau_{s q} a / \mu U$ \\
\hline & $43.63 \%$ & 6 & 0.3941 & 0.1617 & 0.3294 & 0.1598 \\
& $28.27 \%$ & 10 & 0.7656 & 0.1536 & 0.5249 & 0.1559 \\
& & & & & \\
\\
0
\end{tabular}

Table 1: Normalised slip length, $\lambda / a$, and normalised tangential stress due to the bulk flow, $\tau a / \mu U$, for reticulated arrays characterised by different values of the area fraction $\phi_{s}$ and mesh size $L$, for $d / a=7$. The last two columns report the values of $\lambda$ and $\tau$ for a square array having the same solid area fraction of the corresponding reticulated array.

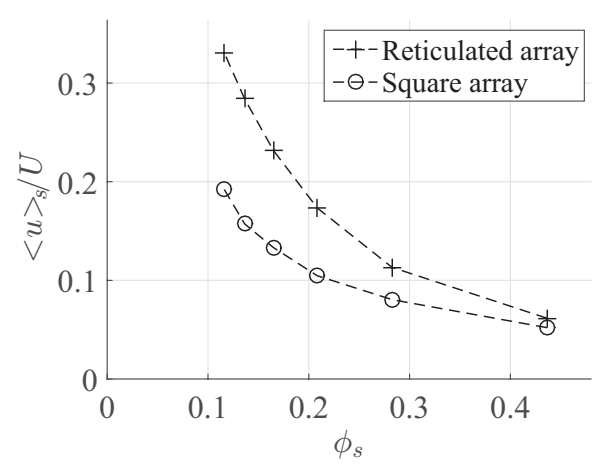

(a)

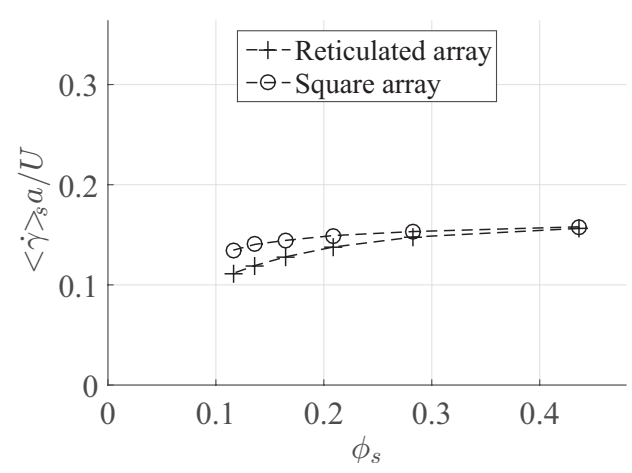

(b)

Figure 12: (a) Normalised slip velocity and (b) normalised bulk shear rate at the interface as a function of solid area fraction for $d / a=7$, comparing square and reticulated arrays. 


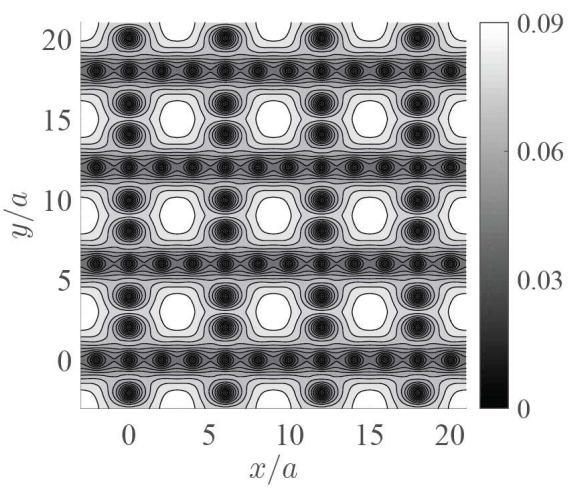

(a)

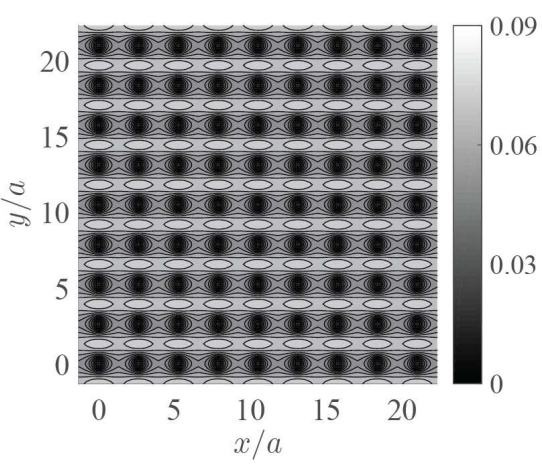

(b)

Figure 13: Contours of normalised streamwise velocity, $u / U$, in the plane $z=a$ for (a) square array and (b) reticulated array. The solid area fraction in (a) and (b) is $\phi_{s}=43.63 \%$ and $d / a=7$.

the ratio of $\langle u\rangle_{s}$ to $\langle\dot{\gamma}\rangle_{s}$. A difference in slip length can therefore be attributed to: i) differences in $\langle u\rangle_{s}$; ii) differences in $\langle\dot{\gamma}\rangle_{s}$; iii) a combined effect of both quantities. Figure 12 (a) compares values of $\langle u\rangle_{s}$ obtained with the reticulated array to those obtained with the square array. The corresponding values of $\langle\dot{\gamma}\rangle_{s}$ are shown in figure 12 (b).

It can be seen that the values of $\langle u\rangle_{s}$ given by the two microstructures are comparable only for relatively large values of $\phi_{s}$. For moderate and small values of $\phi_{s}$, the reticulated array gives a significantly larger value of $\langle u\rangle_{s}$ than the square array. Over a similar range of values of $\phi_{s}$, differences in the values of $\langle\dot{\gamma}\rangle_{s}$ corresponding to the two microstructures are relatively small (figure 12 (b)). The observed dependence of the slip length on the microstructure is thus mainly due to changes in the slip velocity, while changes in the bulk shear rate at the interface play a relatively marginal role.

A question arises as to why the slip velocity is larger in the case of the reticulated array. Comparison of figures $11 \mathrm{a}$ and b shows that for a given area fraction, the reticulated array is characterised by larger connected regions not occupied by particles. Because the flow in these regions is relatively unobstructed by the particles, the slip velocity in these regions could be substantially larger than the slip velocity in the interstices between the particles in the square array case. To verify this hypothesis, we plotted iso-contours of $u$ in the plane $z=a$, comparing the reticulated and square array (figure 13). Characteristic velocities in the open areas bounded by chains in the reticulated array case are at least $50 \%$ larger than those in the interstices between particles in the square array case. These relatively large velocities are spread over regions of linear size comparable to the mesh size $L$. In contrast, in the square array the smaller slip velocities are spread over regions of characteristic linear dimension $\ell \ll L$, where $\ell$ is the average inter-particle separation. We have $\phi_{s}=(L / a-1) \pi a^{2} / L^{2}$ for the reticulated array and $\phi_{s}=\pi a^{2} / \ell^{2}$ for the square array. Therefore the ratio $L^{2} / \ell^{2}$ of the interfacial areas occupied by large-velocity fluid (reticulated array) to that occupied by low-velocity fluid increases with decreasing $\phi_{s}$. Since the slip velocity is an area averaged quantity, larger local velocities spread over larger areas will give a larger value of the slip velocity.

We also note that despite significant differences in slip length between the reticulated array and the square array, the interfacial shear stress is comparable in the two cases (cf. table 1). 


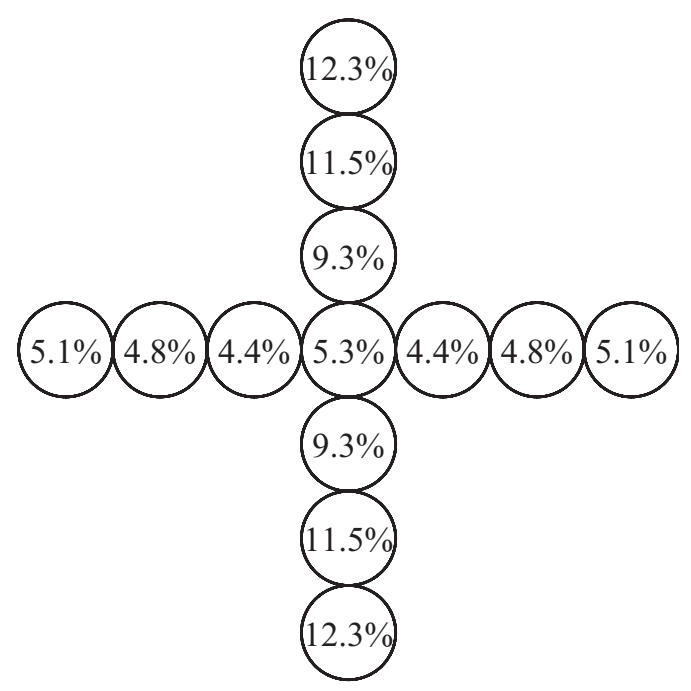

Figure 14: Percentage contribution to the tangential stress due to the bulk flow $\tau$ for each particle in a reticulated array, $\phi_{s}=20.8 \%$ and $d=7 a$. The horizontal chain in the diagram is parallel to the flow direction.

Comparing the values of $\tau$, we note that despite significant differences in slip length between the reticulated array and the square array, the values of the tangential stress due to the bulk flow are comparable in the two cases (cf. table 1).A significant difference between the square and reticulated array is that, while in the square array each particle will contribute equally to $\tau$, in the reticulated array different particles can be subject to different hydrodynamic forces, and therefore the local tangential stress will be in general non-uniform. To characterise the degree of non-uniformity in $\tau$, we show in the diagram of figure 14 the fraction of the total value of $\tau$ contributed by each particle. This fraction is calculated as the ratio of the hydrodynamic force on each particle to the sum of the hydrodynamic forces exerted by all the particles within the computational domain.

The chains of particles arranged perpendicular to the flow direction, or transverse chains, carry the largest contribution to $\tau$. In the case considered in figure 14 the transverse chain contributes to more than $66 \%$ of the total value of $\tau$. The hydrodynamic force acting on the transverse chain is strongly non-uniform: the largest contribution, $12 \%$, is associated to the particles located near the mid point of the transverse chain (farthest away from the intersection point). The central particle, located at the intersection between the longitudinal and transverse chain, contributes to only $5.3 \%$ of the total value of $\tau$. In comparison, particles belonging to the longitudinal chain, oriented along the flow direction, are subject to a relatively uniform bulk tangential stress.

The natural choice of length scale to characterise the flow past the reticulated array is the mesh size $L$. It is therefore expected that the slip length will scale as $\lambda=L f\left(\phi_{s}\right)$, where $f\left(\phi_{s}\right)$ is a function having relatively weak dependence on $\phi_{s}$ (and therefore on $L)$. In figure $15 \mathrm{a}$, the values of $\lambda$ for the reticulated array are plotted as a function $L$. Over the range of values simulated, the slip length is seen to increase only slightly faster than linearly with $L$, confirming a scaling of the type $\lambda \sim L f\left(\phi_{s}\right)$. Davis \& Lauga (2009) carried out an analysis of the slip length for a flat partial slip surface composed of a mesh-like distribution of thin solid regions, obtaining a semi-empirical relation of the 


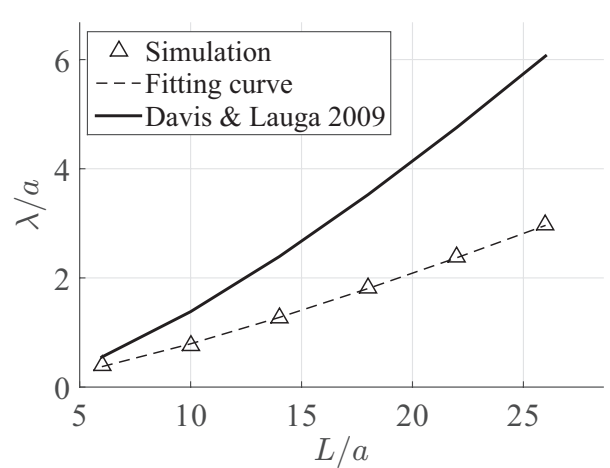

(a)

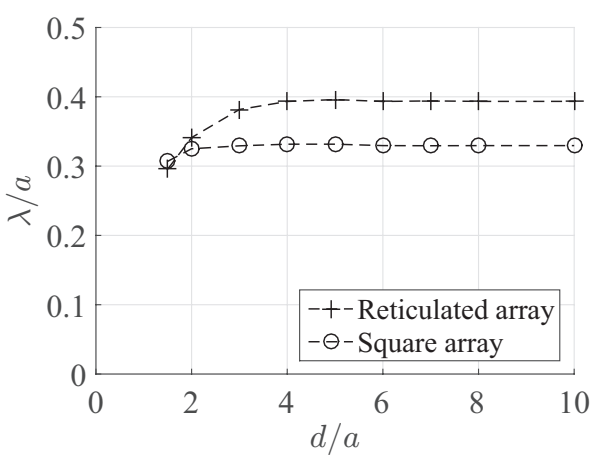

(b)

Figure 15: Normalised slip length vs. (a) normalised mesh size and (b) normalised gap size; reticulated array, $d=7 a$. The area fraction in (b) is $43.63 \%$. The dashed line in figure (a) is equation (3.4) for $A=-0.039$ and $B=0.03$.

form

$$
\frac{\lambda}{L}=A \ln \left(\phi_{s}\right)+B
$$

where $A$ and $B$ are constants. The values obtained by Davis \& Lauga (2009) were $A=-$ 0.107 and $B=0.003$. Fitting the simulation data (dashed line in figure 15a), we obtain $A=-0.039$ and $B=0.03$. As for the square array, the effect of the protuberance of the no-slip region in the liquid gives a larger value of $\lambda$ for a given value of $\phi_{s}$ as compared to a flat partial slip surface. We have attempted a simple rescaling of by a factor $9 \pi / 16$ as done for a square array (see Sec. 3.2) to account for the difference between the results of Davis \& Lauga (2009) and ours, but the results have not been as satisfying as for the square array case. This is due to the fact that for a percolating network hydrodynamic interactions between the particles are important even in the dilute limit. Therefore, the ratio of the hydrodynamic force on a single sphere to that of a single disk cannot represent a good re-scaling factor.

The results above suggest that - in analogy with the case of super-hydrophobic surfaces - different scalings for the slip length hold depending on whether the solid or the liquid are the continuous phases in the plane of the particle-laden interface. In the reticulated network the solid is the continuous phase, and the free-slip interface is the dispersed phase. On the contrary, in the case of the square array, the solid is the dispersed phase. When the solid is dispersed phase and the fluid is the continuous phase in the particleladen interface, the particle radius is the characteristic length scale, and the slip length scales as $\lambda \propto a / \phi_{s}$ (for sufficiently small $\phi_{s}$ ). When the solid is the continuous phase, the mesh size $L$ is the characteristic length, and the influence of the particle radius enters only into a weak - potentially logarithmic - dependence on the solid fraction.

\section{Conclusions}

We have presented numerical simulations for shear flow past a monolayer of neutrallywetting spheres embedded in a liquid-gas interface. In our simulations the shear flow is produced by a flat wall translating parallel to the monolayer. The results are also applicable to situations in which the monolayer translates with respect to a neighbouring 
wall, a situation which occurs for example in certain evaporating droplets problems (Yunker et al. 2011).

Towards extracting effective parameters for the particle-laden interface, we fit the simulation data to the predictions of a Navier slip boundary condition. The simulations provide accurate values for the slip length as a function of the area fraction, the gap size between the monolayer and the wall, and the microstructure of the monolayer.

The dependence of $\lambda$ on $\phi_{s}$ follows scaling laws similar to those developed for shear flow past super-hydrophobic microstructured surfaces: $\lambda \sim a / \phi_{s}$ for a dilute square array, and $\lambda \sim L f\left(\phi_{s}\right)$ where $f\left(\phi_{s}\right)$ is a weak function of $\phi_{s}$ for a reticulated array. The slip length is smaller than for flow past super-hydrophobic surfaces for the same area fraction and microstructure. This feature can be explained by accounting for the fact that most semi-empirical correlations for superhydrophobic surfaces are developed for flat slip/no slip surfaces, while in our case the spheres protrude significantly into the flow. For a square array, a simple prefactor re-scaling based on the ratio of Stokes drag on a sphere to drag on a disk yields good agreement with the simulation data.

For a given area fraction, the slip length for a reticulated array is larger than for a square array. This feature is associated to the presence in a reticulated array of relatively large connected areas unoccupied by particles: the absence of particles "blocking" the flow apparently leads to a larger slip velocity, and consequently to a larger slip length.

The numerical method we employed enabled to accurately compute the tangential stress on the monolayer due to the bulk flow, i.e. the drag force per unit area due to the "subphase" and, for the case of reticulated array, the contribution of each sphere to this quantity. Values for the tangential stress could be useful, for instance, to estimate whether chains of particles would break under the effect of strong hydrodynamic forces. We calculate the tangential stress due to the bulk flow directly from the hydrodynamic force acting on each particle and not from the macroscopic shear rate, as usually done in studies on superhydrophobic surfaces.

In addition to providing insights into the flow in the neighborhood of a sheared monolayer of interfacial colloids, the results of this paper can be useful to estimate effective resistance coefficients for particle-laden interfaces. For instance, the Stokes drag on a spherical particle having a partial slip surface with slip length $\lambda$ is given by $F=6 \pi \mu R V(1+2 \lambda / R) /(1+3 \lambda / R)$, where $R, V$ are the sphere radius and velocity, respectively (Luo \& Pozrikidis 2008). Assuming that partial slip is produced by surfaceactive particles, this expression can be combined with the appropriate expressions for $\lambda$ developed in this paper to calculate the rise velocity of a bubble covered by colloids given the surface coverage by particles or, conversely, the time-dependent surface coverage from the measured bubble velocity.

The slip length is not easily linked to the surface viscosity of the particle-laden interface, as the two parameters model different physical aspects. However, a conceptual link between the two quantities exists. The tangential stress boundary condition (Brenner 2013) states that the tangential stress exerted by the bulk subphase on the particles balances the divergence of the surface stress tensor (the tangential stress on the gas-liquid regions of the particle-laden interface is zero owing to the no-shear condition). The Navier slip equation 1.1 can be interpreted as a linear closure for the tangential stress $\simeq \mu\langle\dot{\gamma}\rangle$ on the monolayer in terms of the particle-fluid velocity difference $\langle u\rangle_{s}$. The surface viscosity and surface elasticity coefficients are instead parameters for the Newtonian closures of the surface stress tensor in terms of the surface deformation rate and surface deformation tensors. The surface viscosity, the surface elasticity, and the slip length therefore enter as parameters in the same differential equation (the tangential stress boundary conditions). However a simple algebraic relation between these effective parameters cannot be found 
in general. The description in terms of slip length complements, other than replace, a description in terms of surface viscosity and surface elasticity.

The slip length approach is expected to be particularly useful when the relation between surface stress tensor and interfacial deformation/deformation rate tensor is not known a priory. For instance, in the analysis of interfacial rheology experiments (see e.g. Buttinoni et al. (2015)) it is often assumed that the drag due to the subphase is negligible. However, rarely estimates for this potentially important quantity are reported. Equation 1.1 can be used to estimate the drag on the monolayer due to the subphase as a function of the average area fraction covered by the particles. The same equation provides a boundary condition for the bulk "subphase" fluid velocity field.

We emphasise that the slip length and the surface viscosity model different micromechanical aspects, and are related to different moments of the hydrodynamic stress on each particle. The surface viscosity is an effective macroscopic property of the particleliquid "mixture" which models the resistance to dilatation and shearing of an element of composite interface (Brenner 2013); the slip length is associated to the locally uniform relative velocity between the particles and the surrounding fluid. The surface viscosity is related to the hydrodynamic torque and stresslet on each particle in the monolayer (Edwards \& Wasan 1991; Lishchuk \& Halliday 2009); the slip length is associated to the hydrodynamic drag on the particles.

The current work, which focuses on a rather idealised situation, can be extended in several directions. In our simulation the relative position between the particles does not change as a result of the flow. This approximation is realistic when the tangential stress due to the bulk flow is much smaller than the gradient in surface pressure (i.e., the two-dimensional pressure due to interparticle forces of non-hydrodynamic origin; see discussion in Gu \& Botto (2016)). Large tangential stresses could lead, after a transient, to a non-uniform particle concentration in the interface. An expected effect, for example, is the compaction of the monolayer in the rear stagnation point of a rising particlecovered bubble. Flow-induced compaction effects on flat interfaces could be simulated with minor modifications of the computational setup used in the current study.

The simulation results presented here are limited to a $90^{\circ}$ contact angle. They are expected to hold as first approximations for contact angles not too different from $90^{\circ}$. Single-particle studies offer some insights into expected trends as a function of the contact angle. Previous work (Danov et al. 2000) has shown that the hydrodynamic drag is larger when the contact angles is such that the particle has a larger protrusion in the liquid phase. For an isolated particle, the drag force on a particle for a flat interface can be expressed as $F=3 \pi \mu a U K\left(\theta_{c}\right)$, where $K\left(\theta_{c}\right)$ is a function of the contact angle for which tables are available (Fischer et al. 2006). According to the scaling laws developed in the current paper, one might expect that $\lambda$ will approximately be proportional to $1 / K$ (at least in the relatively dilute limit): for variations in the contact angle such that the particles will protrude more and more in the liquid phase, we expect a reduction in the slip length. This expected behaviour assumes the the fluid interface between the particles is perfectly flat; fluctuations in the interface due to thermal motion could change this picture quite substantially, as discussed by (Boniello et al. 2015)). For contact angles close to $0^{\circ}$ or $180^{\circ}$ one needs to consider that the area fraction as defined in the current paper is not representative of the surface coverage by the particles, as in these limiting cases the particles barely touch the fluid interface.

While we have started evidencing some effects on the microstructure on the slip length by comparing square arrays and mesh-like particle networks, more realistic microstructures should be investigated. These include the regular hexagonal structure of colloidal crystals at fluid interfaces (Irvine et al. 2010) and the fractal structure of percolating 
networks of aggregated interfacial colloids (Aveyard et al. 2000; Poulichet \& Garbin 2015).

\section{Acknowledgements}

We acknowledge financial support from the European Community through grant no. 618335 "FlowMat: Flow and Capillarity in Materials Science".

\section{REFERENCES}

Aveyard, Robert, Clint, John H, Nees, Dieter \& Paunov, Vesselin N 2000 Compression and structure of monolayers of charged latex particles at air/water and octane/water interfaces. Langmuir 16 (4), 1969-1979.

Batchelor, George Keith 2000 An introduction to fluid dynamics. Cambridge university press.

Binks, Bernard P 2002 Particles as surfactants:similarities and differences. Current Opinion in Colloid 83 Interface Science 7 (1), 21-41.

Binks, Bernard P \& Horozov, Tommy S 2006 Colloidal particles at liquid interfaces. Cambridge University Press.

Bluemink, JJ, Lohse, D, Prosperetti, A \& Van Wijngaarden, L 2008 A sphere in a uniformly rotating or shearing flow. Journal of Fluid Mechanics 600, 201-233.

Bluemink, JJ, Lohse, D, Prosperetti, A \& Van Wijngaarden, L 2010 Drag and lift forces on particles in a rotating flow. Journal of fluid mechanics 643, 1-31.

Boniello, Giuseppe, Blanc, Christophe, Fedorenko, Denys, Medfai, Mayssa, Mbarek, Nadia Ben, In, Martin, Gross, Michel, Stocco, Antonio \& Nobili, Maurizio 2015 Brownian diffusion of a partially wetted colloid. Nature materials 14.

Botto, L \& Prosperetti, A 2012 A fully resolved numerical simulation of turbulent flow past one or several spherical particles. Physics of Fluids (1994-present) 24 (1), 013303.

Bournival, Ghislain, Ata, Seher \& Wanless, Erica J 2015 The roles of particles in multiphase processes: Particles on bubble surfaces. Advances in colloid and interface science 225, 114-133.

BRENNER, HOWARD 2013 Interfacial transport processes and rheology. Elsevier.

Buttinoni, Ivo, Zell, Zachary A, Squires, Todd M \& Isa, Lucio 2015 Colloidal binary mixtures at fluid-fluid interfaces under steady shear: structural, dynamical and mechanical response. Soft matter 11 (42), 8313-8321.

Dani, Archit, Keiser, Geoff, Yeganeh, Mohsen S \& Maldarelli, Charles 2015 Hydrodynamics of particles at an oil-water interface. Langmuir .

Danov, Krassimir, Aust, Richard, Durst, Franz \& Lange, Ulrich 1995 Influence of the surface viscosity on the hydrodynamic resistance and surface diffusivity of a large Brownian particle. Journal of colloid and interface science 175 (1), 36-45.

Danov, Krassimir D, Dimova, Rumiana \& Pouligny, Bernard 2000 Viscous drag of a solid sphere straddling a spherical or flat surface. Physics of Fluids (1994-present) 12 (11), $2711-2722$.

Davis, Anthony MJ \& Lauga, Eric 2009 The friction of a mesh-like super-hydrophobic surface. Physics of Fluids (1994-present) 21 (11), 113101.

Davis, Anthony MJ \& Lauga, ERIC 2010 Hydrodynamic friction of fakir-like superhydrophobic surfaces. Journal of Fluid Mechanics 661, 402-411.

De Gennes, Pierre-Gilles 1985 Wetting: statics and dynamics. Reviews of modern physics $57(3), 827$.

Deemer, Arthur R \& Slattery, John C 1978 Balance equations and structural models for phase interfaces. International Journal of Multiphase Flow 4 (2), 171-192.

Dörr, Aaron \& Hardt, Steffen 2015 Driven particles at fluid interfaces acting as capillary dipoles. Journal of Fluid Mechanics 770, 5-26.

Dörr, Aaron, Hardt, Steffen, Masoud, Hassan \& Stone, Howard A 2016 Drag and diffusion coefficients of a spherical particle attached to a fluid-fluid interface. Journal of Fluid Mechanics 790, 607-618. 
EDWARDS, DA \& WASAN, DT 1991 A micromechanical model of linear surface rheological behavior. Chemical engineering science 46 (5), 1247-1257.

Fischer, Th M, Dhar, P \& Heinig, P 2006 The viscous drag of spheres and filaments moving in membranes or monolayers. Journal of Fluid Mechanics 558, 451-475.

Frijters, Stefan, Günther, Florian \& Harting, Jens 2012 Effects of nanoparticles and surfactant on droplets in shear flow. Soft Matter 8 (24), 6542-6556.

Gu, Chuan \& Botto, Lorenzo 2016 Direct calculation of anisotropic surface stresses during deformation of a particle-covered drop. Soft matter 12 (3), 705-716.

Happel, John \& Brenner, Howard 2012 Low Reynolds number hydrodynamics: with special applications to particulate media, , vol. 1. Springer Science \& Business Media.

Horozov, Tommy S 2008 Foams and foam films stabilised by solid particles. Current Opinion in Colloid 85 Interface Science 13 (3), 134-140.

Hunter, Timothy N, Pugh, Robert J, Franks, George V \& Jameson, Graeme J 2008 The role of particles in stabilising foams and emulsions. Advances in Colloid and Interface Science 137 (2), 57-81.

Irvine, William TM, Vitelli, Vincenzo \& Chaikin, Paul M 2010 Pleats in crystals on curved surfaces. Nature 468 (7326), 947-951.

Kotula, Anthony P \& Anna, Shelley L 2012 Probing timescales for colloidal particle adsorption using slug bubbles in rectangular microchannels. Soft Matter 8 (41), 1075910772 .

Kumar, Avinash, Datta, Subhra \& Kalyanasundaram, Dinesh 2016 Permeability and effective slip in confined flows transverse to wall slippage patterns. Physics of Fluids (1994-present) $\mathbf{2 8}$ (8), 082002.

Lauga, Eric \& Stone, Howard A 2003 Effective slip in pressure-driven Stokes flow. Journal of Fluid Mechanics 489, 55-77.

Lewandowski, Eric P, Cavallaro Jr, Marcello, Botto, lorenzo, Bernate, Jorge C, Garbin, Valeria \& Stebe, Kathleen J 2010 Orientation and self-assembly of cylindrical particles by anisotropic capillary interactions. Langmuir 26 (19), 15142-15154.

LishCHUK, SV \& HALLIDAY, I 2009 Effective surface viscosities of a particle-laden fluid interface. Physical Review E 80 (1), 016306.

Liu, Qianlong \& Prosperetti, Andrea 2011 Pressure-driven flow in a channel with porous walls. Journal of fluid mechanics 679, 77-100.

Luo, Haoxiang \& Pozrikidis, C 2008 Effect of surface slip on Stokes flow past a spherical particle in infinite fluid and near a plane wall. Journal of Engineering Mathematics 62 (1), $1-21$.

Martinez, A Cervantes, Rio, Emmanuelle, Delon, Giles, Saint-Jalmes, Arnaud, Langevin, Dominique \& Binks, Bernard P 2008 On the origin of the remarkable stability of aqueous foams stabilised by nanoparticles: link with microscopic surface properties. Soft Matter 4 (7), 1531-1535.

NG, Chiu-On \& WAng, CY 2009 Stokes shear flow over a grating: implications for superhydrophobic slip. Physics of Fluids (1994-present) 21 (1), 013602.

Petkov, Jordan T, Denkov, Nikolai D, Danov, Krassimir D, Velev, Orlin D, Aust, Richard \& Durst, Franz 1995 Measurement of the drag coefficient of spherical particles attached to fluid interfaces. Journal of colloid and interface science 172 (1), 147-154.

Poulichet, Vincent \& Garbin, Valeria 2015 Ultrafast desorption of colloidal particles from fluid interfaces. Proceedings of the National Academy of Sciences 112 (19), 5932-5937.

PozRIKIDIs, C 2007 Particle motion near and inside an interface. Journal of Fluid Mechanics $\mathbf{5 7 5}, 333-357$.

Rapacchietta, AV \& Neumann, AW 1977 Force and free-energy analyses of small particles at fluid interfaces: II. spheres. Journal of Colloid and Interface Science 59 (3), 555-567.

Rothstein, Jonathan P 2010 Slip on superhydrophobic surfaces. Annual Review of Fluid Mechanics 42, 89-109.

Sbragaglia, Mauro \& Prosperetti, Andrea 2007 A note on the effective slip properties for microchannel flows with ultrahydrophobic surfaces. Physics of Fluids (1994-present) 19 (4), 043603.

Shang, Jianying, Flury, Markus \& Deng, Youjun 2009 Force measurements between 
particles and the air-water interface: Implications for particle mobilization in unsaturated porous media. Water Resources Research 45 (6).

Shelley, SR, Smith, JD, Hibbins, AP, SAmbles, JR \& Horsley, SAR 2016 Fluid mobility over corrugated surfaces in the Stokes regime. Physics of Fluids (1994-present) 28 (8), 083101.

Sierakowski, AdAm J 2016 Gpu-centric resolved-particle disperse two-phase flow simulation using the physalis method. Computer Physics Communications .

Singh, P \& JosePh, DD 2005 Fluid dynamics of floating particles. Journal of Fluid Mechanics $\mathbf{5 3 0}, 31-80$.

Stancik, Edward J, Kouhkan, Mehrnaz \& Fuller, Gerald G 2004 Coalescence of particleladen fluid interfaces. Langmuir 20 (1), 90-94.

Subrahmanyam, TV \& Forssberg, Eric 1988 Froth stability, particle entrainment and drainage in flotationa review. International Journal of Mineral Processing 23 (1), 3353.

Subramaniam, Anand Bala, Abkarian, Manouk \& Stone, Howard A 2005 Controlled assembly of jammed colloidal shells on fluid droplets. Nature materials 4 (7), 553-556.

Tambe, David E \& Sharma, Mukul M 1994 The effect of colloidal particles on fluid-fluid interfacial properties and emulsion stability. Advances in colloid and interface science $\mathbf{5 2}$, $1-63$.

Tsapis, N, Dufresne, ER, Sinha, SS, Riera, CS, Hutchinson, JW, Mahadevan, L \& WeItz, DA 2005 Onset of buckling in drying droplets of colloidal suspensions. Physical Review Letters 94 (1), 018302.

Weber, Martin E, Blanchard, Duncan C \& Syzdek, Lawrence D 1983 The mechanism of scavenging of waterborne bacteria by a rising bubble. Limnology and Oceanography 28 (1), 101-105.

Ybert, Christophe, Barentin, Catherine, Cottin-Bizonne, Cecile, Joseph, Pierre \& BocQuet, LydERIC 2007 Achieving large slip with superhydrophobic surfaces: Scaling laws for generic geometries. Physics of Fluids (1994-present) 19 (12), 123601.

Yunker, Peter J, Still, Tim, Lohr, Matthew A \& Yodh, AG 2011 Suppression of the coffee-ring effect by shape-dependent capillary interactions. Nature 476 (7360), 308-311.

Zhang, Z \& Prosperetti, A 2005 A second-order method for three-dimensional particle simulation. Journal of Computational Physics 210 (1), 292-324. 